\section{Photoelectric Response of Selenium-type Photovoltaic Cells to X-Rays}

A commercratuy available selenium-type photovoltaic cell was irradiated with $\mathbf{X}$-rays produced with a commercial type of low-voltage X-ray tube with a thermionic cathode and an inclined copper target. Lead covers with circular apertures of different diameters were placed over the photocell to alter the flux of X-rays on the surface of the cell at a constant $\mathbf{X}$-radiation intensity, and the $\mathrm{X}$-radiation intensity was varied by changing the anode current of the $X$-ray tube. Fig. 1 shows a family of curves representing the photocell current, $I_{p}$, as a function of the $X$-ray tube anode current, $I_{a}$, with the anode voltage, $V_{a}$, as parameter, the curves having been obtained with a distance of $25 \mathrm{~mm}$. between the photosensitive surface of the cell and the centre of the target of the X-ray tube, $17.5 \mathrm{~mm}$. of the distance being in the vacuum space of the tube, $1.5 \mathrm{~mm}$. the thickness of its eylindrical envelope of soda-lime glass, and the remainder of the air space between the tube and the photocell surface. The curves marked $A$ refer to an aperture of $240 \mathrm{~mm} .{ }^{2}$ in the lesd cover, and those marked $B$ refer to a $60-\mathrm{mm} .^{2}$ aperture.

The characteristics of Fig. 1 indicate a linear relation between photoelectric response and X-radiation flux irrespective of whether the flux is changed

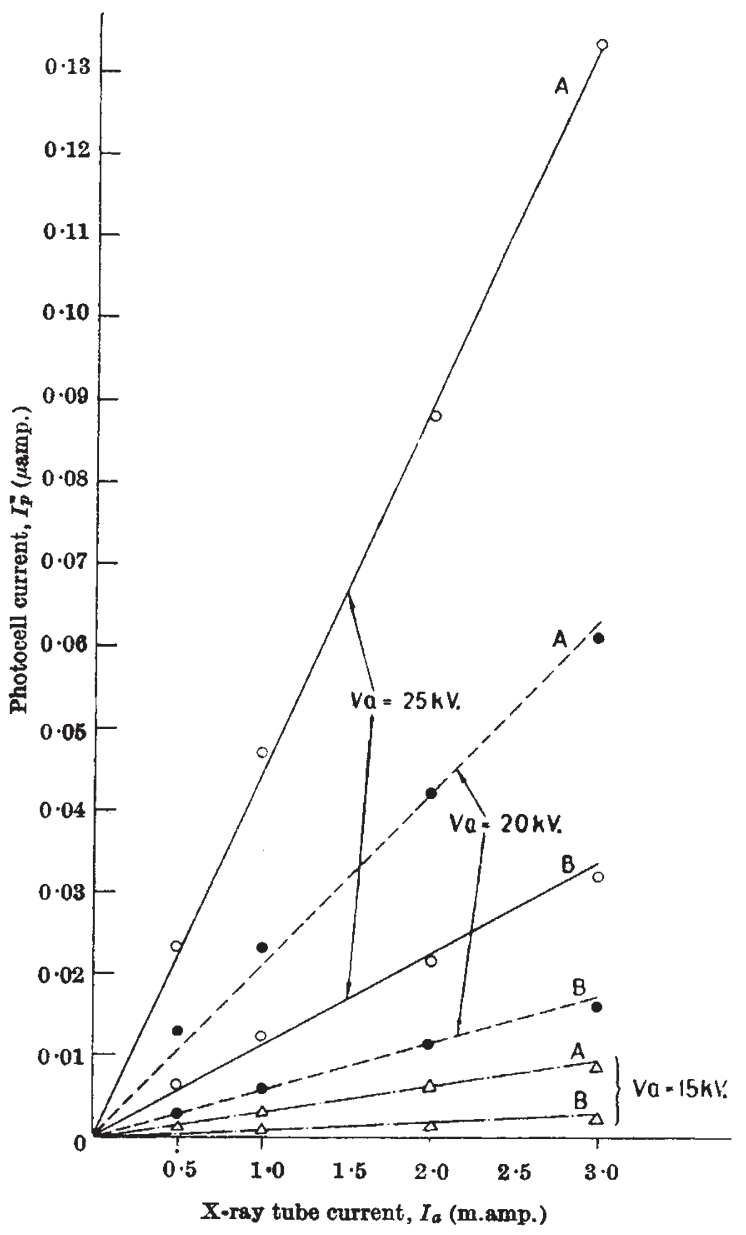

Fig. 1. Curves $A$, irradiation area, $240 \mathrm{~mm} .^{2}$; curves $B$, irradiation area, $60 \mathrm{~mm}$. $^{\mathrm{s}}$ by altering the $\mathrm{X}$-radiation intensity or by varying the size of the area of irradiation.

Increasing the aperture of the lead cover over the photocell to a diameter of $25 \mathrm{~mm}$., compared with $17.5 \mathrm{~mm}$. for obtaining the curves $A$ in Fig. 1, demonstrated the reduction of radiation intensity towards the boundary of the X-ray beam. The photocurrent response indicated that the X-radiation flux increased only by 50 per cent, whereas it would have doubled if the $\mathrm{X}$-radiation intensity had been uniform over the whole area of the aperture.

A second photocell of the same type geve a similar photoelectric response characteristic, but all photocurrent values were reduced to one-third of those of Fig. 1, although the two cells had the same photoelectric sensitivity to light. The fact that both cells were equally sensitive to light but differently sensitive to X-rays may probably be interpreted in terms of light being effective only at the surface of the photosensitive layer and $X$-rays being effective in its depth, and that there was a difference in the thickness and/or the structure of the photosensitive layers of the two cells. The depth effect of X-rays may possibly in part also account for the dependence of the photoelectric response on the anode voltage of the $\mathrm{X}$-ray tube.

Ferranti, Ltd., at present

\section{R. Fernberg}

Electrical Engineering Department, Manchester College of Science and Technology. Feb. 24.

\section{Plasticity of Graphite}

THE creep behaviour of graphite in the temperature range $1,300-2,000^{\circ} \mathrm{C}$. has been examined for the first time. The experiments were carried out by applying loads to cantilevers heated in vacuo by the passage of an electric current and to spring specimens heated in a high-temperature high-vacuum furnace. The specimens were made in the laboratory by extruding a wide range of raw materials (pitch coke, petroleum coke, lamp black, cellulose and natural graphite). Springs of rectangular section were also machined from commercially manufactured materials (reactor graphite and commercial electrode graphite). The apparatus and techniques used in these experiments were similar to those used in dynamic experiments previously described ${ }^{1}$.

It was found that, for a wide range of temperatures, raw materials, specimen geometries, flexural and torsional stresses, the creep deformation could be statistically fitted within the limits of experimental error to the function :

$$
D_{t}=A+k \log _{10} t+B t
$$

where $D_{t}$ is strain at time $t$ and $t$ is time (min.).

$A, k$ and $B$ are constants for each experiment. Similarly, the creep recovery on removing the load conformed to the function:

$$
D_{t^{1}}=A^{1}+k^{1} \log t
$$

A typical creep curve is shown as a linearized plot in Fig. $1 a$. Fig. $1 b$ is a plot of the deviations of the experimental points from the function determined by the method of least squares.

The coefficients $k$ and $B$ were linearly dependent on the applied stress at constant temperature and were related to the absolute temperature as follows : 